Pulsar Astronomy - 2000 and Beyond

ASP Conference Series, Vol. 202, 2000

M. Kramer, N. Wex, and R. Wielebinski, eds.

\title{
Timing of the young pulsar $\mathrm{J} 1907+0918$
}

K. Xilouris

Center Development Lab, NRAO, Charlottesville, VA 22903, USA

D.R. Lorimer, A. Dowd

Arecibo Observatory, HC3 Box 53995, Arecibo, PR 00612, USA

\begin{abstract}
We have extensively searched for periodic signals in the area of the soft gamma-ray repeater SGR $1900+14$, at 430 and $1410 \mathrm{MHz}$ with the Arecibo telescope. Our observations did not reveal the 5.16-s periodicity reported for the magnetar down to a $430-\mathrm{MHz}$ flux density limit of $150 \mu \mathrm{Jy}$. During the search we discovered a 226 -ms radio pulsar, PSR J1907+0918. The period derivative implies the characteristic age for this pulsar is only $38 \mathrm{kyr}$. Independent evidence in support of this apparent youth are the unusually high degree of circular polarization and its relatively flat radio spectrum. The close proximity of PSR J1907+0918 to SGR $1900+14$ suggests that they may have evolved from a massive binary system. Regardless of this possibility, it is presently not clear to us that SGR $1900+14$ is associated with the supernova remnant G42.8+0.6.
\end{abstract}

\section{The search for radio pulsations from SGR $1900+14$}

In early June 1998 we observed the soft gamma-ray repeater SGR $1900+14$ using the Arecibo telescope seven days after the source became active following a long period of quiescence. The search for radio pulsations at $430 \mathrm{MHz}$ and 1.4 $\mathrm{GHz}$ was carried out with the Penn State Pulsar Machine (PSPM), a filterbank which records the total power outputs of the receiver over $128 \times 60 \mathrm{kHz}$ frequency channels every $80 \mu \mathrm{s}$.

Our search did not reveal the 5.16 -s period reported for SGR $1900+14$ by Kouveliotou et al. (1998). Based on our observations we place an upper limit of approximately $150 \mu \mathrm{Jy}$ to the flux density of the magnetar at frequencies around $430 \mathrm{MHz}$. Following the announcement of a low-frequency detection of this pulsar by Shitov (see contribution elsewhere in these proceedings) we observed the magnetar using the $47 \mathrm{MHz}$ dipole feed. Although this system could detect B0950+08 and B0823+26 we were unable to detect the magnetar. Further, more sensitive, low-frequency Arecibo observations would be worthwhile.

The $1410-\mathrm{MHz}$ observations did, however, reveal the presence of a very promising 113-ms pulsar candidate with a dispersion measure of $350 \mathrm{~cm}^{-3} \mathrm{pc}$. Subsequent observations made around the end of September both at Arecibo and Effelsberg, confirmed the existence of the pulsar (PSR J1907+0918) and identified its true period to be $226 \mathrm{~ms}$ (Xilouris et al. 1998, IAUC No. 7023). 


\section{Timing observations of PSR $1907+0918$}

Follow-up timing results show that PSR J1907+0918 is an interesting radio pulsar in its own right. Regular timing observations using the PSPM were initiated in mid October 1998. A standard TEMPO analysis of pulse time-of-arrival measurements spanning a 9-month baseline yields the following timing solution: R.A. (J2000) 19 h $07 \mathrm{~m} 22.4 \mathrm{sec}$, Dec. 09' 18' 31.8", $P=0.226106270831 \mathrm{sec}$, $\dot{P}=94.286 \times 10^{-15}$, these parameters apply to the reference MJD 51216 . Uncertainties for each parameter are one unit of the least significant digit quoted. Current post-fit residuals are $98.6 \mu \mathrm{s}$. In spite of present covariances between position and $\dot{P}$, it is clear from high-precision period measurements over the 9 -month baseline that the quoted $\dot{P}$ is correct. The characteristic age is $38 \mathrm{kyr}$ and implied dipole surface magnetic field is $4.7 \times 10^{12} \mathrm{G}$.

\section{Discussion}

Apart from globular cluster pulsars, PSR J1907+0918 and SGR $1900+14$ are the closest pair of neutron stars in the sky that do not presently consitute a binary. The angular separation between them is $\sim 2$ arcmin. An assumed distance of $5 \mathrm{kpc}$ implies a spatial separation of $3.2 \mathrm{pc}$, while a distance of $7 \mathrm{kpc}$ implies a separation of $4.5 \mathrm{pc}$. Either this close proximity is simply a coincidence, or the neutron stars both originated from a disrupted massive binary system. Such scenarios have been invoked to explain the proximity of the Crab pulsar to B0525+21 (Gott, Gunn \& Ostriker, 1970, ApJ 160, L91) and PSR B1853+01 and PSR B1854+00 (Wolzczan Cordes \& Dewey 1991, ApJ, 372, L99).

Regardless of the fact that these two neutron stars may have had a common origin, we would like to point out that it is presently not clear to us whether the nearby supernova remnant G42.8+0.6 is associated with SGR 1900+14 (which has an estimated age of $10 \mathrm{kyr}$; Kouveliotou et al. 1999, ApJ, 510 L115) or PSR J1907+0918 (with a characteristic age of $38 \mathrm{kyr}$ ). As mentioned above, Shitov has recently detected 5.16-s pulsations from SGR $1900+14$ at $100 \mathrm{MHz}$ and determined a dispersion measure of $281.4 \mathrm{~cm}^{-3} \mathrm{pc}$. Based on this and the dispersion measure for $\mathrm{J} 1907+0918$, both these neutron stars are at a comparable distance from the Earth $(5-7 \mathrm{kpc})$. If PSR J1907+0918 is associated with G42.8+0.6 then the spatial separation between them is $20 \mathrm{pc}$ (assuming a distance to the remnant of $5 \mathrm{kpc}$ ) or $28 \mathrm{pc}$ (assuming a distance to the remnant of $7 \mathrm{kpc}$ ). The transverse velocity required for the remnant and the pulsar to be associated is then between $550-760 \mathrm{~km} \mathrm{~s}^{-1}$.

It should also be noted that, since this region of the Galactic plane has a high density of supernova remnants and pulsars, it is possible that neither PSR J1907+0918 nor SGR 1900+14 have any connection with G42.8+0.6. Future VLBI proper motion measurements of PSR J1907+0918, perhaps using Arecibo-Effelsberg-GBT, would certainly help to clarify this situation.

Acknowledgments. We wish to thank A. Wolszczan and D. Backer for providing access to their datataking equipment and hence making these observations possible. We would also like to thank F. Camilo and I. Stairs for useful discussions concerning the Arecibo timing observations. Arecibo Observatory is run by Cornell University under contract with the National Science Foundation. 